\title{
Anomalous Time Scaling of the Mean Square Distance in On-Off Diffusion
}

\author{
$\operatorname{AUTHOR}(\mathrm{S})$ :
}

Miyazaki, Syuji

\section{CITATION:}

Miyazaki, Syuji. Anomalous Time Scaling of the Mean Square Distance in On-Off Diffusion. Journal of the Physical Society of Japan 2000, 69(9): 2719-2722

\section{ISSUE DATE:}

2000-09-15

URL:

http://hdl.handle.net/2433/254367

\section{RIGHT:}

(C2000 The Physical Society of Japan; この論文は出版社版でありませ ん。引用の際には出版社版をご確認ご利用ください。; This is not the published version. Please cite only the published version. 


\title{
Anomalous Time Scaling of the Mean Square Distance in On-Off Diffusion
}

\author{
Syuji MIYAZAKI \\ Department of Applied Analysis and Complex Dynamical Systems, Graduate School of Informatics, \\ Kyoto University, Kyoto 606-8501
}

\begin{abstract}
The mean square distance $\sigma^{2}(t)$ of the diffusion induced by on-off intermittency is derived based on the continuous-time random walk theory. It obeys a scaling law $\sigma^{2}(t)=2 D t \phi(t / \tau)$ with diffusion constant $D$ and characteristic time $\tau$, which is confirmed by the use of numerical iterations of a specific periodic map. The scaling function $\phi$ and the power spectrum of the on-off intermittency variable $I(\omega)$ are analytically obtained from the distribution function of the laminar duration. Normal diffusion $(\phi(z) \sim 1)$ and slow diffusion $(\phi(z) \sim 1 / \sqrt{z})$ are observed, respectively, for $t \gg \tau$ and $t \ll \tau)$. The former and latter correspond to the flat part $(I(\omega) \sim$ const $)$ and the power law $(I(\omega) \sim 1 / \sqrt{\omega})$, respectively, for the power spectrum.
\end{abstract}

KEYWORDS: continuous-time random walk, first passage time, on-off intermittency, on-off diffusion

Many studies have been reported on the phenomenon of on-off intermittency which is typically observed when a synchronized chaotic motion in a coupled oscillator system becomes unstable. ${ }^{1,2)}$ On-off intermittency has been observed not only in numerical models ${ }^{3-5)}$ but also in laboratory experiments. ${ }^{6-11)}$ Recently, Ott and Sommerer proposed a new dissipative dynamical model showing onoff intermittency. ${ }^{12}$ ) In their model, on-off intermittency is observed when a specific one-dimensional motion loses its stability and two-dimensional motion sets in. Furthermore, Lai and Grebogi ${ }^{13)}$ extended the OttSommerer model in such a way that two equivalent subspaces show physically equivalent 1d motion. Namely, this model has two symmetric invariant subspaces. As the control parameter of the system is varied, the largest Lyapunov exponent of the motion transverse to the invariant subspaces changes its sign from negative to positive. When this transverse Lyapunov exponent is positive, the state point shows an intermittent switching motion between the invariant subspaces which is similar to on-off intermittency. This is termed two-state, on-off intermittency.

Harada, Hata, and Fujisaka ${ }^{14}$ ) extended the LaiGrebogi model for two-state on-off intermittency in such a way that the system exhibits an infinite-state on-off intermittency by utilizing a periodic potential. The property of the potential to be periodic implies the possibility of a diffusive behavior of a particle. They termed this phenomenon on-off diffusion.

On the other hand, Geisel and Thomae considered one-dimensional periodic maps, the elements of which generate intermittent chaos, to show that the maps cause anomalous diffusion characterized by the nonlinear growth of the mean square deviation. ${ }^{15)}$ On-off diffusion can be also modeled by a periodic map, as we shall see later.

The diffusion process discussed by Geisel and Thomae and the on-off diffusion we consider have many common features. The trajectory remains long in a cell, i.e., in one of the elements of the periodic map, and then jumps to its neighboring cell. The intra-cell motion and the jumping motion alternate in an irregular manner, which can be reduced to a one-dimensional random walk with a waiting time during the intra-cell motion.

Thus, the continuous-time random walk theory proves to be very helpful in analyzing such correlated diffusions. ${ }^{16)}$ Let $\sigma^{2}(t)=\left\langle\Delta x^{2}(t)\right\rangle$ be the mean square displacement, the Laplace-transform of which is given by

$$
\tilde{\sigma}^{2}(s)=\mathcal{L}\left[\sigma^{2}(t)\right]=\frac{\tilde{\psi}(s)}{s-s \tilde{\psi}(s)},
$$

where $\tilde{\psi}(s)$ denotes the Laplace-transform of the probability density distribution $\psi(t)$ of the waiting time $t$ in the cell: $\tilde{\psi}(s)=\mathcal{L}[\psi(t)]$. In the case of on-off diffusion, the distribution $\psi(t)$ can be regarded as that of the laminar duration of the on-off intermittency.

Yamada and Fujisaka ${ }^{17)}$ analyzed the on-off intermittency using a multiplicative noise process:

$$
l(t)=\left(\lambda_{\perp}+\xi(t)\right) l-l^{3},
$$

where $\xi(t)$ denotes Gaussian white noise with a vanishing average. This Langevin equation has a stable equilibrium solution $l=0$ for $\lambda_{\perp}<0$. On the other hand, the equilibrium solution becomes unstable for $\lambda_{\perp}>0$. When $\lambda_{\perp}$ is slightly greater than 0 , numerical solutions of the Langevin equation yield such temporal evolutions as on-off intermittency. The laminar duration is measured according to the interval between the neighboring intersecting points between the time series $l(t)$ and the threshold $l=l_{0}$. In the vicinity of the critical point $\lambda_{\perp}=0$, the nonlinear term can be neglected, so that the distribution function of the first passage time with the start $l=l_{c}$ and with the goal $l=l_{0}\left(l_{c}<l_{0}\right)$ is analytically obtained as follows: ${ }^{18)}$

$$
\psi(t)=\frac{c}{\sqrt{4 \pi D_{\perp}}} t^{-3 / 2} \exp \left[-\frac{\left(\lambda_{\perp} t-c\right)^{2}}{4 \pi D_{\perp}}\right],
$$


where $D_{\perp}$ denotes half of the variance of the transverse local expansion rates whose average is equal to $\lambda_{\perp}$, and $c=\log \left(l_{0} / l_{c}\right)$. Here we approximate the temporal evolution in such a way that the amplitude $l(t)$ which reaches the threshold $l=l_{0}$ is suddenly reinjected to the same place $l=l_{c}$, and that the amplitude $l(t)$ increases again with fluctuation. This distribution function involves the power law $\psi(t) \propto t^{-3 / 2}$, which is one of the most remarkable characteristics of the on-off intermittency. ${ }^{19)}$ The average laminar duration is given by

$$
\bar{t}=\int_{0}^{\infty} t \psi(t) d t=\frac{c}{\lambda_{\perp}}
$$

When we compare $\psi(t)$ with experimental data, we should first determine $\bar{t}$ for a fixed threshold, as well as $\lambda_{\perp}$ and $D_{\perp}$. The three parameters $\lambda_{\perp}, D_{\perp}$, and $c=\lambda_{\perp} \bar{t}$ of $\psi(t)$ are determined without ambiguity.

The Laplace-transform of the above-mentioned $\psi(t)$ is given by

$$
\tilde{\psi}(s)=\mathrm{e}^{\alpha[1-\sqrt{1+4 \tau s}]},
$$

where $\alpha=\frac{\lambda_{\perp}}{2 D_{\perp}} c=\frac{\bar{t}}{2 \tau}$ and $\tau=\frac{D_{\perp}}{\lambda_{\perp}^{2}}$. For $\tau s \ll 1$ or $1 \ll \tau s \ll$ $\frac{1}{\alpha^{2}}$, we can approximate $\tilde{\psi}(s)$ as

$$
\tilde{\psi}(s)=1+\alpha[1-\sqrt{1+4 \tau s}],
$$

so that

$$
\begin{aligned}
\mathcal{L}\left[\sigma^{2}(t)\right] & =\frac{\tilde{\psi}(s)}{s-s \tilde{\psi}(s)}, \\
& \approx \frac{1}{4 \alpha \tau s^{2}}+\frac{\sqrt{1+4 \tau s}}{4 \alpha \tau s^{2}}-\frac{1}{s}, \\
& \propto \begin{cases}s^{-2} & (\tau s \ll 1), \\
s^{-3 / 2} & \left(1 \ll \tau s \ll \frac{1}{\alpha^{2}}\right) .\end{cases}
\end{aligned}
$$

Hence, we have

$$
\sigma^{2}(t)= \begin{cases}\frac{1}{4 \alpha} \frac{t}{\tau} & (t \gg \tau), \\ \frac{1}{\sqrt{\pi} \alpha} \sqrt{\frac{t}{\tau}} & \left(\alpha^{2} \tau \ll t \ll \tau\right) .\end{cases}
$$

The mean square deviation is given by the inverse Laplace-transform as

$$
\begin{aligned}
\sigma^{2}(t) & =\mathcal{L}^{-1}\left[\frac{1}{4 \alpha \tau s^{2}}+\frac{\sqrt{1+4 \tau s}}{4 \alpha \tau s^{2}}-\frac{1}{s}\right], \\
& =\frac{t}{4 \alpha \tau}+\frac{2}{\alpha \sqrt{\pi}} \sqrt{\frac{t}{4 \tau}} \mathrm{e}^{-\frac{t}{4 \tau}} M\left(2, \frac{3}{2}, \frac{t}{4 \tau}\right)-1,
\end{aligned}
$$

where $M$ denotes the Kummer ${ }^{20)}$ Since the following asymptotic form holds ${ }^{20)}$

$$
\begin{aligned}
M\left(2, \frac{3}{2}, z\right) \rightarrow \quad & \frac{\sqrt{\pi}}{2} \mathrm{e}^{z} \sqrt{z}\left(1+O\left(|z|^{-1}\right)\right) \\
& \text { for } z \rightarrow \infty,
\end{aligned}
$$

we obtain $\sigma^{2}(t) \rightarrow 2 D t$ for $t \rightarrow \infty$ with $D=\frac{1}{4 \alpha \tau}=\frac{\lambda_{\perp}}{2 c}$, so that we have the following scaling relation

$$
\frac{\sigma^{2}(t)}{2 D t}=\phi\left(\frac{t}{\tau}\right)
$$

with the scaling function

$$
\begin{aligned}
\phi(z)= & \frac{1}{2}+\sqrt{\frac{4}{\pi z}} \mathrm{e}^{-z / 4} M\left(2, \frac{3}{2}, \frac{z}{4}\right) \\
& \begin{cases}=1 & (z \gg 1), \\
\propto \frac{1}{\sqrt{z}} & \left(\alpha^{2} \ll z \ll 1\right) .\end{cases}
\end{aligned}
$$

For $\alpha^{2} \tau \ll t \ll \tau$, the time series of on-off intermittency has a self-similar structure. ${ }^{21)}$ The physical origin of the power law $\phi(z) \propto 1 / \sqrt{z}$ is this fact.

We confirm the scaling relation $\frac{\sigma^{2}(t)}{2 D t}=\phi\left(\frac{t}{\tau}\right)$ by a numerical simulation using the following piecewise linear map:

$$
\begin{aligned}
X_{t+1} & = \begin{cases}\frac{X_{t}}{a} & \left(0 \leq X_{t}<a\right), \\
\frac{1-X_{t}}{1-a} & \left(a \leq X_{t}<1\right),\end{cases} \\
x_{t+1} & =f\left(x_{t}\right) \\
& = \begin{cases}b^{-2} x_{t} & \left(0 \leq x_{t}<b^{2}, 0 \leq X_{t}<a\right), \\
b x_{t} & \left(0 \leq x_{t}<b^{2}, a \leq X_{t}<1\right), \\
\frac{b-x_{t}}{b(1-b)}-1 & \left(b^{2} \leq x_{t}<b\right), \\
\frac{x_{t}-b}{1-b}+1 & \left(b \leq x_{t}<1\right),\end{cases}
\end{aligned}
$$

where $f\left(x_{t}\right)$ is periodic in $x_{t}$,

$$
f\left(x_{t}+k\right)=f\left(x_{t}\right)+k, \quad(k=0, \pm 1, \pm 2, \cdots) .
$$

This map is a generalization of the map introduced by Hata and Miyazaki to describe the on-off intermittency, ${ }^{22)}$ where the reinjection branches are replaced by the branches causing the jump to the neighboring cell.

We term the unit interval $[k, k+1)$ the $k$-th cell hereafter. When $x_{t}$ falls in the interval $\left[b^{2}+k, b+k\right)$ belonging to the $k$-th cell, $x_{t+1}$ jumps to the $(k-1)$-th cell. In the same way, $x_{t+1}$ jumps to the $(k+1)$-th cell when $x_{t} \in[b+k, 1+k)$. Thus, diffusive motion occurs.

Following Grossmann and Fujisaka, ${ }^{23)}$ we decompose $x_{t}$ into the integer part $N_{t}=\left[x_{t}\right]$ and the fractional part $y_{t}: x_{t}=N_{t}+y_{t}$. The map $f$ becomes $N_{t+1}+y_{t+1}=N_{t}+f\left(y_{t}\right)$, which is then decomposed into

$$
\begin{aligned}
N_{t+1}-N_{t} & =\left[f\left(y_{t}\right)\right] \equiv \Delta\left(y_{t}\right), \\
y_{t+1} & =f\left(y_{t}\right)-\left[f\left(y_{t}\right)\right] \equiv g\left(y_{t}\right),
\end{aligned}
$$

so that $N_{t}=\sum_{n=0}^{t-1} \Delta\left(y_{n}\right)$ for $N_{0}=0$.

The mean square distance is given by

$$
\left\langle N_{t}\right\rangle=\sum_{j, k}^{t-1}\left\langle\Delta\left(y_{j}\right) \Delta\left(y_{k}\right)\right\rangle,
$$

where the angle brackets denote the average for all initial conditions $y_{0}$. We select the parameter $b$ of the map as a function of $a$ in such a way that no drift motion appears, namely $\left\langle N_{t}\right\rangle=0$. We have, therefore,

$$
\frac{\left\langle N_{t}^{2}\right\rangle}{t}=\frac{1}{t} \sum_{k=0}^{t-1}\left\langle\Delta\left(y_{k}\right)^{2}\right\rangle \approx \int d y \rho(y) \Delta^{2}(y),
$$

for $t \gg \tau$, where $\tau$ is the characteristic correlation time of the map $g$, and $\rho(y)$ is an invariant density of $g$ satisfying 


$$
\rho(y)=\int d x \delta[g(x)-y] \rho(x),
$$

which is explicitly given as a step function by Hata and Miyazaki, ${ }^{22)}$ so that $\left\langle N_{t}^{2}\right\rangle=2 D t$ for $t \gg \tau$ with a diffusion constant $D=\frac{1}{2} \int d y \rho(y) \Delta^{2}(y)$, which can be evaluated analytically in our case.

On the other hand, the characteristic time $\tau$ can also be obtained analytically. The transverse Lyapunov exponent $\lambda_{\perp}$ and its variance $2 D_{\perp}$ are given by

$$
\begin{aligned}
\lambda_{\perp} & =(1-3 a) \log b, \\
2 D_{\perp} & =9 a(1-a)(\log b)^{2},
\end{aligned}
$$

with $a=a_{c}(1+\epsilon)$. As $\epsilon \rightarrow 0, \lambda_{\perp} \propto \epsilon, D_{\perp} \sim$ const, $\bar{t} \propto \epsilon^{-1}$, and $\tau \propto \epsilon^{-2}$, so that we have $\alpha^{2} \tau=\frac{\bar{t}^{2}}{2 \tau}=O(1)$. The critical point is given by $a_{c}=\frac{1}{3}$, at which on-off intermittency occurs in the map $g$, and on-off diffusion occurs in the periodic map. ${ }^{22)}$

We obtain the mean square distance $\left\langle N_{t}^{2}\right\rangle$ from the numerical iteration of the periodic map, and plot $\frac{\left\langle N_{t}^{2}\right\rangle}{2 D t}$ versus $\frac{t}{\tau}$ for $\epsilon=0.0005(\times), 0.001(\square), 0.003(\circ), 0.005(\cdot)$, and $0.01(\diamond)$ with the theoretical curve in Fig. 1. A good agreement between the theory and the numerical experiment is observed over four decades of $t / \tau$.

The scaling law obeyed by the mean square distance can be reproduced by the following scaling assumption. Assume the scaling form $\left\langle N_{t}^{2}\right\rangle=\epsilon^{\delta} \phi\left(\epsilon^{\beta} t\right)$, with the scaling function

$$
\phi(z) \propto \begin{cases}z^{-\gamma} & (0<z \ll 1), \\ \text { const } & (z \gg 1) .\end{cases}
$$

As we mentioned previously, on-off intermittency has the characteristic time $\tau \propto \epsilon^{-2}$, so that we have $\beta=2$. For $t \gg \tau$, normal diffusion is observed, and therefore, we have $D \propto \epsilon^{\delta}$. For $t \ll \tau$, it follows that $\left\langle N_{t}^{2}\right\rangle=\epsilon^{\delta-2 \gamma} t^{-\gamma}$. Assume that this does not depend on $\epsilon$, and we have $\delta-2 \gamma=0$. In the case of the periodic map which we considered above, the diffusion constant is proportional to $\epsilon$, so that we have $\delta=1$. Hence, we obtain $\gamma=1 / 2$, which is consistent with the asymptotic form of the theoretically derived scaling function.

The characteristic time $\tau=\frac{D_{\perp}}{\lambda_{\perp}^{2}}$ appears in the scaling law of the power spectrum: ${ }^{24)}$

$$
I(\omega)=h / \sqrt{1+\sqrt{1+(4 \tau \omega)^{2}}},
$$

with $h \approx 0.263046$. A similar expression was derived by Fujisaka and Yamada for the generalized power spectrum $I_{q}(\omega){ }^{25)}$ This scaling form can also be derived from $\psi(t)$ as follows. The correlation function for the discrete variable $l_{n}$ :

$$
C(m)=\lim _{N \rightarrow \infty} \frac{1}{N} \sum_{m=0}^{N} l_{n+m} l_{n},
$$

is related to the power spectrum:

$$
I(\omega) \propto \lim _{N \rightarrow \infty} \frac{1}{N} \sum_{m=0}^{N} \cos (m \omega) C(m),
$$

via the Wiener-Khinchine theorem. Following Schus- scaling function

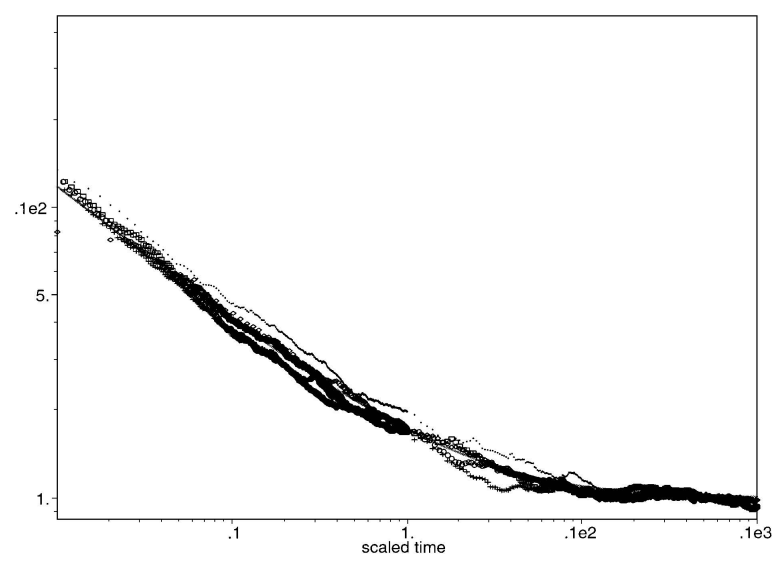

Fig. 1. Scaling function $\phi$ vs scaled time $t / \tau$.

ter, ${ }^{26)} C(m)$ can be expressed with $\psi(m)$ as

$$
C(m)=\sum_{k=0}^{m} C(m-k) \psi(k)+\delta_{m, 0},
$$

where $\psi(0)=0$ and $C(0)=1$. The Laplace-transform of both sides yields

$$
\tilde{C}(s)=\frac{1}{1-\tilde{\psi}(s)}
$$

so that we have

$$
\begin{aligned}
I(\omega) & =\int_{0}^{\infty} d t \cos (\omega t) C(t), \\
& =\frac{1}{2}\left[\frac{1}{1-\tilde{\psi}(\mathrm{i} \omega)}+\frac{1}{1-\tilde{\psi}(-\mathrm{i} \omega)}\right],
\end{aligned}
$$

where we pass on to the continuous time variable. For $\tau s \ll 1$ or $1 \ll \tau s \ll 1 / \alpha^{2}, \psi(s)$ can be approximated as $1+\alpha(1-\sqrt{4 \tau s})$. Substituting this approximation into the above expression, we obtain

$$
I(\omega)=\frac{1}{\sqrt{2} \alpha} \frac{1}{\sqrt{1+\sqrt{1+(4 \tau \omega)^{2}}}}
$$

It should be noted that $\tau s \ll 1(t \gg \tau)$ and $1 \ll$ $\tau s \ll 1 / \alpha^{2}\left(\alpha^{2} \tau \ll t \ll \tau\right)$ correspond to the flat region $I(\omega) \sim$ const and the power-law (self-similar) region $I(\omega) \propto 1 / \sqrt{\omega}$, respectively. Thus it is proved that the characteristic time $\tau$ separates the two time regions. Aside from the numerical factor, the above expression $I(\omega)$ coincides with the scaling form previously obtained by Miyazaki and Hata for a piecewise linear map ${ }^{24)}$ as well as by Fujisaka and Yamada for a multiplicative noise process. $^{25)}$ This surprising result may imply that the scaling function is universal. The validity and the foundation of this universality are left for future study.

The author is indebted to S. Grossmann for careful reading of the manuscript and critical comments, and to T. Harada for providing relevant references. The author also acknowledges valuable discussions with $\mathrm{H}$. Fujisaka and H. Hata. This study was partially financed by a Grand-in-Aid for Scientific Research (C) of the Japan Society for the Promotion of Science. 
1) H. Fujisaka and T. Yamada: Prog. Theor. Phys. 74 (1985) 918.

2) H. Fujisaka and T. Yamada: Prog. Theor. Phys. 75 (1986) 1087.

3) J. Redondo, E. Roldan and G. J. de Valcarcel: Phys. Lett. A 210 (1996) 301.

4) M. Sauer and F. Kaiser: Phys. Rev. E 54 (1996) 2468.

5) H. Tominaga, H. Fujisaka and W. Just: J. Phys. Soc. Jpn. 66 (1997) 3406.

6) T. Yamada, K. Fukushima and T. Yazaki: Prog. Theor. Phys. Suppl. 99 (1989) 120

7) P. W. Hammer, N. Platt, S. M. Hammel, J. F. Heagy and B. D. Lee: Phys. Rev. Lett. 73 (1994) 1095

8) F. Rodelsperger, A. Cenys and H. Benner: Phys. Rev. Lett. 75 (1996) 2594

9) Y. H. Yu, K. Kwak and T. K. Lim: Phys. Lett. A 198 (1995) 34

10) A. Cenys, A. Namajunas, A. Tamasevicius and T. Schneider: Phys. Lett. A 213 (1996) 259.

11) T. John, R. Stannarius and U. Behn: Phys. Rev. Lett. 83 (1999) 749 .

12) E. Ott and J. C. Sommerer: Phys. Lett. A 188 (1994) 39.
13) Y. C. Lai and C. Grebogi: Phys. Rev. E 52 (1995) R3313.

14) T. Harada, H. Hata and H. Fujisaka: J. Phys. A 32 (1999) 1557.

15) T. Geisel and S. Thomae: Phys. Rev. Lett. 52 (1984) 1936.

16) E. W. Montroll and H. Scher: J. Stat. Phys. 9 (1973) 101.

17) T. Yamada and H. Fujisaka: Prog. Theor. Phys. 76 (1986) 582.

18) L. M. Ricciardi: Diffusion Processes and Related Topics in Biology, Lecture Notes in Biomathematics 14 (Springer, Berlin, 1977) p. 504

19) J. F. Heagy, N. Platt and S. M. Hammel: Phys. Rev. E 49 (1994) 1140.

20) M. Abramowitz and I. A. Stegun: Handbook of Mathematical Functions (Dover, New York, 1965) p. 61.

21) H. Fujisaka and T. Yamada: Prog. Theor. Phys. 77 (1987) 1045.

22) H. Hata and S. Miyazaki: Phys. Rev. E 55 (1997) 5311.

23) S. Grossmann and H. Fujisaka: Phys. Rev. A 26 (1982) 1779.

24) S. Miyazaki and H. Hata: Phys. Rev. E 58 (1998) 7172.

25) H. Fujisaka and T. Yamada: Prog. Theor. Phys. 90 (1993) 529.

26) H. G. Schuster: Deterministic Chaos (VCH, Weinheim, 1988) p. 94. 